\title{
Cytokine Switch and Bystander Suppression of Autoimmune Responses to Multiple Antigens in Experimental Autoimmune Encephalomyelitis by a Single Recombinant T-Cell Receptor Ligand
}

\author{
Sushmita Sinha, ${ }^{1,2}$ Sandhya Subramanian, ${ }^{1,2}$ Lisa Miller, ${ }^{1,2}$ Thomas M. Proctor,,${ }^{1,6}$ Chris Roberts, ${ }^{1,2}$ \\ Gregory G. Burrows, ${ }^{2,3,6}$ Arthur A. Vandenbark, ${ }^{1,2,4,6}$ and Halina Offner ${ }^{1,2,5,6}$ \\ ${ }^{1}$ Neuroimmunology Research, Veterans Affairs Medical Center, and Departments of ${ }^{2}$ Neurology, ${ }^{3}$ Biochemistry and Molecular Biology, ${ }^{4}$ Molecular \\ Microbiology and Immunology, and ${ }^{5}$ Anesthesiology and Perioperative Medicine and ${ }^{6}$ Tykeson Multiple Sclerosis Research Laboratory, Oregon Health \& \\ Science University, Portland, Oregon 97239
}

Recombinant T-cell receptor ligands (RTLs) can reverse clinical and histological signs of experimental autoimmune encephalomyelitis (EAE) in an antigen-specific manner, and are currently in clinical trials for treatment of subjects with multiple sclerosis (MS). Antigen specificity of RTL raises the question as to whether this treatment would be successful in MS patients where target antigens are unknown. Using spinal cord homogenate or combinations of two different peptides to induce disease, we found that treatment with single RTL could reverse EAE as long as targeted T-cells were present. Therapy with three different RTLs each caused a significant reduction in IL-17 and increases in IL-10 and IL-13 in peptide-activated splenocytes, reduced proliferation of both cognate and bystander specificities of lymph node cells, and reduced inflammatory lesions and secreted IL-17 and IL-2 from peptide-activated spinal cord cells. These results show that treatment with single RTLs can induce a cytokine switch in cognate T-cells that inhibits both the target and bystander T-cells, providing new evidence for the potential applicability of RTL therapy in MS.

\section{Introduction}

Recombinant T-cell receptor (TCR) ligands (RTLs) containing the membrane distal $\alpha 1+\beta 1$ domains of class II major histocompatibility complex (MHC) molecules linked covalently to specific peptides can be used to regulate $\mathrm{T}$-cell responses and inhibit clinical experimental autoimmune encephalomyelitis (EAE). RTLs were shown to signal directly through the TCR as a partial agonist, and could prevent and treat active or passive myelin basic protein (MBP)-induced monophasic EAE in Lewis rats (Burrows et al., 1998; Wang et al., 2003), myelin oligodendrocyte glycoprotein (MOG)-induced chronic EAE in HLA-DR2 transgenic mice (Vandenbark et al., 2003; Chou et al., 2004; Link et al., 2007), proteolipid protein (PLP)-induced relapsing EAE in SJL/J mice (Huan et al., 2004), and MOG-35-55-induced chronic EAE

Received Dec. 5, 2008; revised Jan. 30, 2009; accepted Feb. 17, 2009.

Dr. Sinha is a Postdoctoral Fellow of the National Multiple Sclerosis Society, and this work was supported in part by National Multiple Sclerosis Society Postdoctoral Fellowship FG1749-A-1 and Grants RG3794A and RG3468; National Institutes of Health Grants NS47661, A143960, NS41965, and NS46877; The Nancy Davis MS Center Without Walls; and the Biomedical Laboratory R\&D Service, Department of Veterans Affairs. We thank Eva Niehaus for assistance in preparing the manuscript.

H.O., G.G.B., A.A.V., and Oregon Health \& Science University (OHSU) have a significant financial interest in Artielle ImmunoTherapeutics, a company that may have a commercial interest in the results of this research and technology. This potential conflict of interest has been reviewed and managed by the $\mathrm{OHSU}$ and Veterans Affairs Medical Center Conflict of Interest in Research Committees.

Correspondence should be addressed to Dr. Halina Offner, Neuroimmunology Research R\&D-31, Portland Veterans Affairs Medical Center, 3710 SW US Veterans Hospital Road, Portland, 0R 97239. E-mail: offnerva@ohsu.edu. D0I:10.1523/JNEUROSCI.5812-08.2009

Copyright $\odot 2009$ Society for Neuroscience $\quad 0270-6474 / 09 / 293816-08 \$ 15.00 / 0$ in C57BL/6 mice (Sinha et al., 2007). RTL constructs derived from DR2 inhibited activation and secretion of proinflammatory cytokines, and also induced IL-10 secretion in human DR2restricted T-cell clones specific for MBP-85-99 or cABL peptides (Burrows et al., 2001; Chang et al., 2001). One such DR2 construct, RTL1000, containing the MOG-35-55 peptide, is currently in a phase 1 safety trial in multiple sclerosis (MS) subjects.

The applicability of antigen-specific therapies for MS is challenging due to lack of a single obvious target antigen and the possible involvement of multiple myelin-reactive CD4 and CD8 $\mathrm{T}$-cell specificities in the inflammatory phase of disease (McFarland and Martin, 2007). Studies using RTL therapy for EAE thus far have demonstrated peptide-specific effects on clinical and histological EAE (Burrows et al., 1998; Vandenbark et al., 2003; Huan et al., 2004; Offner et al., 2005; Sinha et al., 2007). That is, EAE induced with a given encephalitogenic determinant can only be treated with RTLs containing the same (cognate) but not a different (noncognate) peptide. To evaluate the potential of RTL treatment for MS patients, it is important to address the issue of whether a single RTL can affect T-cells with multiple specificities. We here address this issue by using single RTL constructs to treat EAE induced with whole spinal cord extracts or multiple encephalitogenic peptides. The results demonstrate that a single RTL can successfully treat ongoing EAE induced with a mixture of encephalitogenic determinants by switching the profile of cytokines secreted by the targeted $\mathrm{T}$-cell specificity from inflammatory to anti-inflammatory factors that can inhibit bystander T-cells with 
a different TCR specificity. Thus, a single RTL construct can effectively treat disease induced by multiple T-cell specificities, provided that the cognate $\mathrm{T}$-cell specificity is present.

\section{Materials and Methods}

Animals. SJL/J female mice were obtained from The Jackson Laboratory at 7-8 weeks of age. The mice were housed in the Animal Resource Facility at the Portland Veterans Affairs Medical Center (Portland, OR) in accordance with institutional guidelines. The study was conducted in accordance with National Institutes of Health guidelines for the use of experimental animals, and the protocols were approved by the Institutional Animal Care and Use Committee.

RTL402 and RTL403 constructions. General methods for the design, cloning, and expression of RTL (including RTL401 used in this study) have been described previously (Burrows et al., 1999; Huan et al., 2004; Offner et al., 2005), and were used in the production of two new RTL constructs for use in mice expressing I-A ${ }^{\mathrm{s}}$, RTL402 (I-A ${ }^{\mathrm{s}}$ moiety with covalently attached PLP-178-191 peptide) and RTL403 (I-A ${ }^{\text {s }}$ moiety with covalently attached MBP-84-104 peptide). In brief, mRNA was isolated from the splenocytes of SJL mice using an Oligotex Direct mRNA mini kit (Qiagen). cDNA encoding the antigen-binding/TCR recognition domain of murine MHC class II I-A ${ }^{\mathrm{s}} \beta$ and $\alpha$ chains was derived from mRNA using two pairs of PCR primers. The two chains were sequentially linked by DNA encoding an amino acid linker (GGQDD) in a two-step PCR, with NcoI and XhoI restriction sites added to the 5' and 3' ends, respectively. Additional sequence encoding the PLP-178-191 (C183S) peptide (NTWTTSQSIAFPSK) and a flexible linker (GGGGSLVPRGSGGGG) was added to the $5^{\prime}$ end of the synthetic gene encoding RTL400 to form RTL402. The same technique was used to link sequence encoding the MBP-84-104 peptide (VHFFKNIVTPRTPPPSQGKGR) to RTL400 to form RTL403. The murine I-A ${ }^{\mathrm{s}}$-derived peptide- $\beta 1 \alpha 1$ genes were ligated into pET21d (+) vector and transformed into Nova blue Escherichia coli host (Novagen) for positive colony selection and sequence verification. RTL402 and RTL403 plasmid constructs were then transformed into E. coli strain BL21 (DE3) expression host (Novagen). The purification of RTL proteins has been described previously (Chang et al., 2001; Huan et al., 2004). The final yield of purified protein varied between 15 and $30 \mathrm{mg}$ per liter of bacterial cell culture.

Immunization with whole spinal cord homogenate. Syngenic whole spinal cord homogenate (WSCH) was prepared as described previously (Veräjänkorva et al., 2002). Briefly, spinal cords from SJL/J mice were removed, mixed with an equal volume of distilled water, and homogenized. Mice were injected subcutaneously in the flanks with $0.1 \mathrm{ml}$ of emulsion consisting of an equal volume of $1 \mathrm{mg} / \mathrm{ml} \mathrm{WSCH}$ (reconstituted in PBS) and CFA containing $4 \mathrm{mg} / \mathrm{ml}$ heat-killed Mycobacterium tuberculosis H37RA on days 0 and 7. Mice also received $200 \mathrm{ng}$ of pertussis toxin (Ptx) intraperitoneally on days 0 and 2. The mice were assessed for signs of EAE according to the following scale: 0 , normal; 1, limp tail or mild hindlimb weakness; 2 , moderate hindlimb weakness or mild ataxia; 3 , moderately severe hindlimb weakness; 4 , severe hindlimb weakness or mild forelimb weakness or moderate ataxia; 5 , paraplegia with no more than moderate forelimb weakness; and 6, paraplegia with severe forelimb weakness or severe ataxia or moribund condition. At the onset of clinical signs of EAE (days 10-11 when the clinical scores were $\geq 2$ ), mice were divided into two groups and treated with vehicle (100 $\mu \mathrm{l}$ of $20 \mathrm{~mm}$ Tris$\mathrm{HCl}, \mathrm{pH}$ 8.6) or with $100 \mu \mathrm{l}$ of $1 \mathrm{mg} / \mathrm{ml}$ RTL401 (containing PLP-139151 peptide) subcutaneously for $8 \mathrm{~d}$. Mice were monitored for changes in disease score until they were killed for ex vivo analyses.

Immunizations with peptides. For the mixed peptide immunization experiments, SJL/J mice were inoculated in the flanks with $0.2 \mathrm{ml}$ of emulsion with the following combinations of peptides: $100 \mu \mathrm{g}$ of PLP$139-151+100 \mu \mathrm{g}$ of PLP-178-191 and an equal volume of CFA containing $2 \mathrm{mg} / \mathrm{ml}$ heat-killed Mycobacterium tuberculosis; $200 \mu \mathrm{g}$ of MBP84-104/CFA (containing $2 \mathrm{mg} / \mathrm{ml}$ heat-killed Mycobacterium tuberculosis) on day 0 and $150 \mu \mathrm{g}$ of PLP-139-151 $+200 \mu \mathrm{g}$ of MBP84-104/CFA (containing $2 \mathrm{mg} / \mathrm{ml}$ heat-killed Mycobacterium tuberculosis) on day 7. Mice were also injected with $200 \mathrm{ng}$ of Ptx intraperitoneally on days 7 and 9 relative to immunization with PLP-139-151 + MBP84-104 peptides. Mice were scored as described above, and at the onset of clinical signs of EAE (when the clinical scores were $\geq 2$ ), mice were divided into three groups and treated with $100 \mu \mathrm{l}$ of $20 \mathrm{~mm}$ Tris- $\mathrm{HCl}$ as controls or with $100 \mu \mathrm{l} \mathrm{of} 1 \mathrm{mg} / \mathrm{ml}$ RTL401 (with covalently attached PLP-139-151 peptide), RTL402 (with PLP-178-191 peptide), or RTL403 (with MBP-84-104 peptide) subcutaneously for $8 \mathrm{~d}$. Mice were monitored for changes in disease score and were boosted with additional RTL treatments as indicated until they were killed for ex vivo analyses. Mice were also given antihistamine injections when they were boosted with vehicle or RTL treatments as a precautionary measure to prevent any hypersensitivity reaction.

Proliferation assays. Draining lymph nodes (LNs) were harvested from vehicle- and RTL-treated mice on day 39 (for WSCH experiment) and day 26 (for double peptide immunization experiments). A single-cell suspension was prepared by homogenizing the tissue through a fine mesh screen. Cells were cultured in a 96-well flat-bottom tissue culture plate at $4 \times 10^{5}$ cells/well in stimulation medium either alone (control) or with indicated test antigens (PLP-139-151, PLP-178-191, and MBP-84-104 peptides) at varying concentrations. The cells obtained from mice immunized with syngenic whole spinal cord were subjected to a panel of antigens as indicated. Cells were incubated for $3 \mathrm{~d}$ at $37^{\circ} \mathrm{C}$ in $7 \% \mathrm{CO}_{2}$. Cells were then pulsed with $0.5 \mu \mathrm{Ci}$ of [ methyl ${ }^{3} \mathrm{H}$ ] thymidine (PerkinElmer) for the final $18 \mathrm{~h}$ of incubation and were harvested onto glass fiber filters, and tritiated thymidine uptake was measured by a liquid scintillation counter. Means and SDs were calculated from triplicate wells. The stimulation index (SI) was calculated by dividing Ag-induced counts per minute $(\mathrm{cpm})$ by medium control cpm.

Cytokine determination by Luminex Bio-Plex assay kit. Single-cell suspensions of spleens from vehicle and RTL treatment groups, at the end of the experiments, were prepared by homogenizing the tissue through a fine mesh screen. Four million cells, from each vehicle- and RTL-treated group, were cultured in the presence of indicated peptides in a 24-well tissue culture plate for $48 \mathrm{~h}$. Culture supernatants were assessed for cytokine levels using a Luminex Bio-Plex cytokine assay kit (Bio-Rad Laboratories) following the manufacturer's instructions. The following cytokines were determined in a single assay in three (for WSCH) or two (for double peptide immunization) separate experiments: IL- $1 \beta$, IFN $\gamma$, TNF $\alpha$, IL-2, IL-4, IL-10, IL-17, IL-5, IL-6, IL-12, and IL-13.

Histopathology. At the termination of experiment, 4 mice from vehicleand RTL-treated groups were perfused with $0.9 \%$ saline followed by cold $4 \%$ paraformaldehyde. Spinal cords were removed and postfixed in $4 \%$ paraformaldehyde for $48 \mathrm{~h}$. Spinal cords were dissected after fixation and embedded in paraffin before sectioning. The sections were stained with luxol fast blue/ periodic acid-Schiff-hematoxylin to assess demyelination and inflammatory lesions, and analyzed by light microscopy.

Statistical analyses. Mean values from each experiment were compared statistically. Differences in group daily clinical scores, peak scores, and cumulative disease index (CDI) were evaluated by the Kruskal-Wallis test followed by the Mann-Whitney test; incidence was evaluated by Fisher's exact test; disease onset was compared by one-way ANOVA followed by Newman-Keuls test; cytokine secretion and T-cell proliferation data were compared by unpaired Student's $t$ test. Data are represented as mean \pm SD.

\section{Results}

In previous studies, we demonstrated that RTLs containing encephalitogenic peptides linked to the outer two domains of the restricting $\mathrm{MHC}$ class II molecules could reverse clinical signs of EAE (Huan et al., 2004; Offner et al., 2005; Sinha et al., 2007). However, the efficacy of RTL treatment for MS may be affected by whether or not a single RTL can suppress encephalitogenic potential of multiple T-cell specificities.

Inhibition of EAE by RTL401 in mice immunized with WSCH We have demonstrated that RTL treatment can successfully treat EAE induced with cognate peptides. Thus, RTL401 can reverse clinical signs of PLP-139-151 peptide-induced EAE in SJL/J 


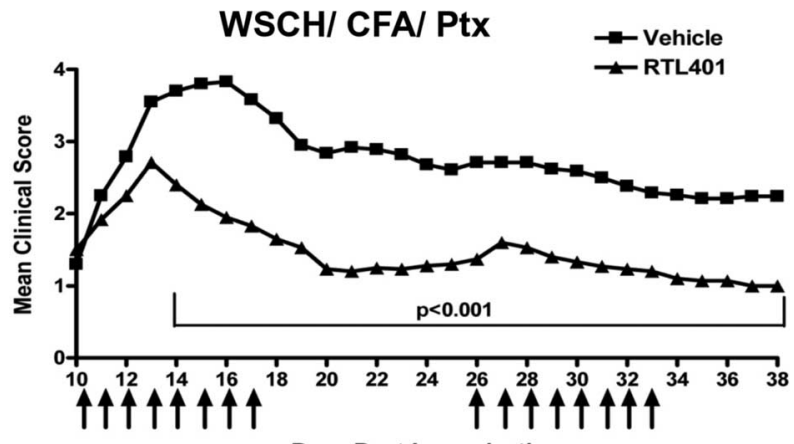

Days Post Immunization

\begin{tabular}{ccccc}
\hline Group & Incidence & Onset & Peak & CDI \\
\hline Vehicle & $20 / 20$ & $11.6 \pm 1.2$ & $4.2 \pm 0.5$ & $71.7 \pm 18.5$ \\
RTL401 & $20 / 20$ & $11.7 \pm 1.0$ & $2.9 \pm 1.0^{\mathrm{a}}$ & $37.2 \pm 22.4^{\mathrm{a}}$ \\
\hline
\end{tabular}

avehicle vs. RTL, $p<0.01$

Figure 1. Subcutaneous administration of RTL 401 treats EAE in SJL/J mice immunized with WSCH/CFA/Ptx. At disease onset (day 10), mice were treated daily with vehicle (Tris $\mathrm{HCl}$, pH 8.5) or $0.1 \mathrm{mg}$ of RTL 401 for $8 \mathrm{~d}$. Mice also received booster injections of $0.1 \mathrm{mg}$ of RTL 401 for $8 \mathrm{~d}$ as indicated in the figure and were scored as outlined in Materials and Methods. Data presented are the mean of three experiments for each group, with 6-8 mice per group. Significant differences between control and treated groups were determined using Mann-Whitney test.

mice; RTL551, MOG-35-55 peptide-induced EAE in C57BL/6 mice; and RTL342M, MOG-35-55-induced EAE in HLA-DR2 Tg mice (Huan et al., 2004; Link et al., 2007; Sinha et al., 2007). However, successful treatment in MS patients, in which the identity and number of encephalitogenic target antigens are unknown, will likely depend on whether a single RTL can influence multiple T-cell specificities. We addressed this issue first by immunizing mice with syngenic whole cord homogenate, which would contain all the relevant myelin antigens, followed by induction of EAE with multiple epitopes and treating mice with single RTL at onset of clinical disease. For WSCH experiments, mice were immunized with the indicated dose of WSCH, and at onset of clinical signs of actively induced EAE (day 10), mice were treated with RTL401 subcutaneously for $8 \mathrm{~d}$. The RTL treatment was very effective in reducing the clinical severity of EAE and arresting disease progression within $4 \mathrm{~d}$ after initiating treatment. Eight days after first treatment completion, mice were boosted with an additional eight consecutive doses of RTL401 and were further observed for 5 more days before killing them on day 39 after immunization (Fig. 1). At the end of the experiment, vehicle-treated mice showed a CDI of $71.7 \pm 18.5$, whereas the RTL-treated mice had a significantly reduced CDI of $37.2 \pm 22.4$ $(p<0.01)$ (Fig. 1). The peak disease score of RTL401-treated mice also was significantly lower than vehicle-treated mice (vehicle vs RTL, $4.2 \pm 0.5$ vs $2.9 \pm 1.0 ; p<0.01$ ) (Fig. 1 ). The therapeutic effect of RTL401 was highly reproducible in three separate experiments. As indicated in Figure $2 A$, panels of 11 and 8 peptides were used to test the proliferative response in cells isolated from LNs and CNS, respectively. Cells from lymph node and CNS showed proliferative responses only to PLP-139-151 peptide in the vehicle as well as the RTL401-treated group. In addition to PLP-139-151, cells isolated from the CNS of vehicletreated mice also proliferated to PLP-43-64. Furthermore, cells from CNS of RTL-treated mice showed a significantly reduced proliferation to PLP-139-151 peptide. Supernatants from splenocytes from RTL401-treated mice stimulated by WSCH in vitro for $48 \mathrm{~h}$ had significantly decreased production of IL-17 and
TNF $\alpha$ compared with spleen cells supernatants from vehicletreated mice (Fig. $2 B$ ). On the other hand, anti-inflammatory cytokines, IL-4, IL-10, and IL-13, were significantly increased in the RTL401-treated group (Fig. 2B), suggesting existence of a strong anti-inflammatory environment in the spleens of RTL401-treated mice.

\section{Reversal of clinical EAE with two new RTL constructs} designed for use in SJL/J mice

Having established treatment of EAE with RTL401 in mice immunized with WSCH, we studied the treatment effect in the mice immunized with different combinations of known encephalitogenic peptides in the SJL/J model of relapsing remitting EAE. We constructed two new RTL molecules, RTL402 (I-A ${ }^{\text {s }}$ moiety linked covalently to PLP-178-191 peptide) and RTL403 (I-A ${ }^{\text {s }}$ linked to MBP-84-104 peptide). We first tested the treatment efficacy of these new RTL individually in PLP-178-191 and MBP-84-104 peptide-induced EAE. Thymic antigens PLP-178191 and MBP-84-104 (Anderson et al., 2000) were able to induce EAE with comparable severity as nonthymic antigen PLP-139151 (Fig. 3). Figure 3, $A$ and $B$, shows that both RTL402 and RTL403 can effectively reverse clinical signs of EAE induced with the respective cognate peptides. The peak disease score and CDI of RTL402- and RTL403-treated mice were significantly lower than in vehicle-treated mice (Fig. $3 A, B$ ). However, neither RTL402 nor RTL403 could significantly affect EAE scores in mice immunized with PLP-139-151/CFA, and RTL401 did not affect EAE induced with PLP-178-191/CFA or MBP-84-104/CFA/Ptx (data not shown), indicating specificity of RTL treatment for the immunizing peptide. LN cells from RTL402- and RTL403treated mice showed reduced proliferation when stimulated with respective cognate peptides (data not shown). Similar to RTL401-treated PLP-139-151-immunized mice, splenocytes from RTL402- and RTL403-treated mice showed reduced production of IL-17 and IFN $\gamma$. In addition to IL-10, we also found increased production of IL-4 from RTL-treated splenocytes obtained from mice immunized with a nonthymic antigen such as PLP-178-191 or MBP-84-104 (data not shown).

\section{Individual RTL treatment effectively reverses EAE induced} with two different peptides

For double peptide immunization experiments, the concentration of the immunizing peptides was adjusted such that immune responses in the lymph nodes could be discerned to each immunizing peptide. Individual RTL401 and RTL402 constructs or RTL401 and RTL403 constructs were used to treat EAE induced with PLP-139-151 + PLP-178-191 or PLP-139-151 + MBP84-104 peptides, respectively. As shown in Figure 4A, RTL401 or RTL402 treatment each reduced the peak severity of the first clinical episode (vehicle vs RTL401, $3.6 \pm 0.6$ vs $2.4 \pm 0.9, p<$ 0.01 ; vehicle vs RTL402, $3.6 \pm 0.6$ vs $2.1 \pm 1.1, p<0.01)$ and CDI (vehicle vs RTL401, $46.3 \pm 8.6$ vs $33.0 \pm 10.9, p<0.01$; vehicle vs RTL402, $46.3 \pm 8.6$ vs $28.1 \pm 14.7, p<0.01)$ in mice immunized with PLP-139-151 + PLP-178-191 peptides. Similarly, RTL401 or RTL403 treatment each were effective in reducing peak disease (vehicle vs RTL401, $2.9 \pm 0.9$ vs $1.0 \pm 0.7, p<0.001$; vehicle vs RTL403, $2.9 \pm 0.9$ vs $1.6 \pm 0.9, p<0.001$ ) and CDI (vehicle vs RTL401, 46.6 \pm 13.2 vs $19.1 \pm 12.6, p<0.001$; vehicle vs RTL403, $46.6 \pm 13.2$ vs $25.4 \pm 15.8, p<0.001)$ of EAE induced with PLP-139-151 + MBP-84-104 peptides (Fig. 4 B). In both experiments, eight consecutive treatments with RTL reduced the mean clinical scores to $\sim 2$. However, in each experiment, a few of the mice in the RTL-treated group began to relapse and therefore all 
A. Proliferative response to myelin antigens
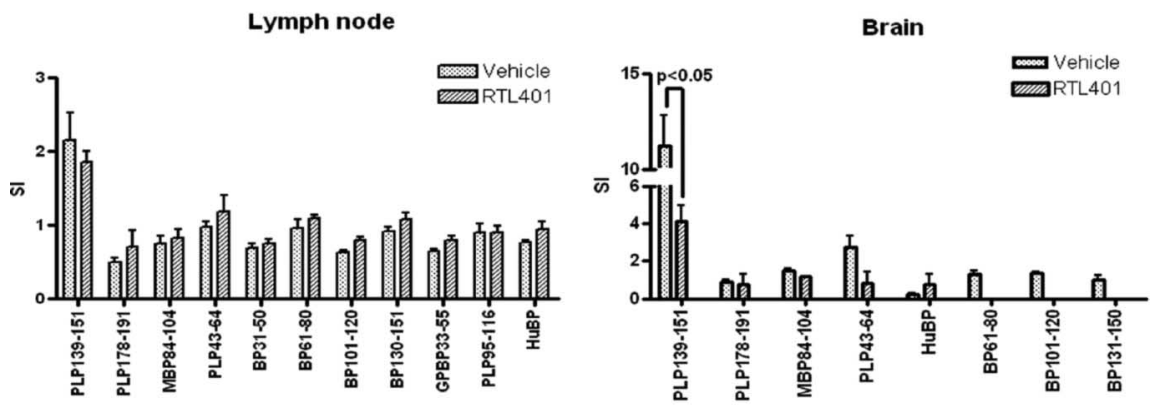

B. Cytokine production in spleen

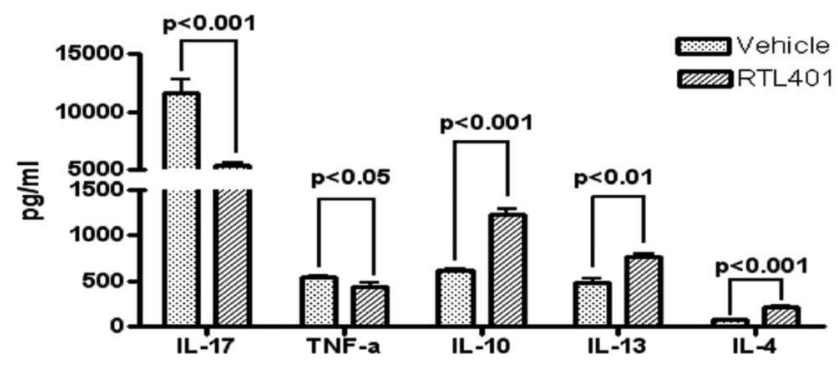

Figure 2. A, Cells from lymph nodes and CNS were isolated from vehicle- and RTL401-treated mice on day 39 after immunization with WSCH. Cells from four representative mice were pooled and T-cell responses were measured by proliferation to the panel of antigens as indicated in the figure, after $72 \mathrm{~h}$ of incubation in stimulation medium, the last $18 \mathrm{~h}$ in the presence of ${ }^{3} \mathrm{H}$-thymidine. Data are presented as stimulation indices $(\mathrm{Ag} \mathrm{cpm} / \mathrm{control} \mathrm{cpm})$ and are representative of three separate experiments. $\boldsymbol{B}$, SJL mice were killed $39 \mathrm{~d}$ after immunization with WSCH. Splenocytes were harvested and cultured in vitro with $10 \mu \mathrm{g}$ of syngenic WSCH. Supernatants were harvested after $48 \mathrm{~h}$ and assayed for cytokine production by using Bio-Plex cytokine assay kit, as described in Materials and Methods. Significant differences between control and experimental groups were determined using Student's $t$ test. Data are presented as the mean \pm SD of three replicate cultures from pooled cells, and are representative of three experiments.
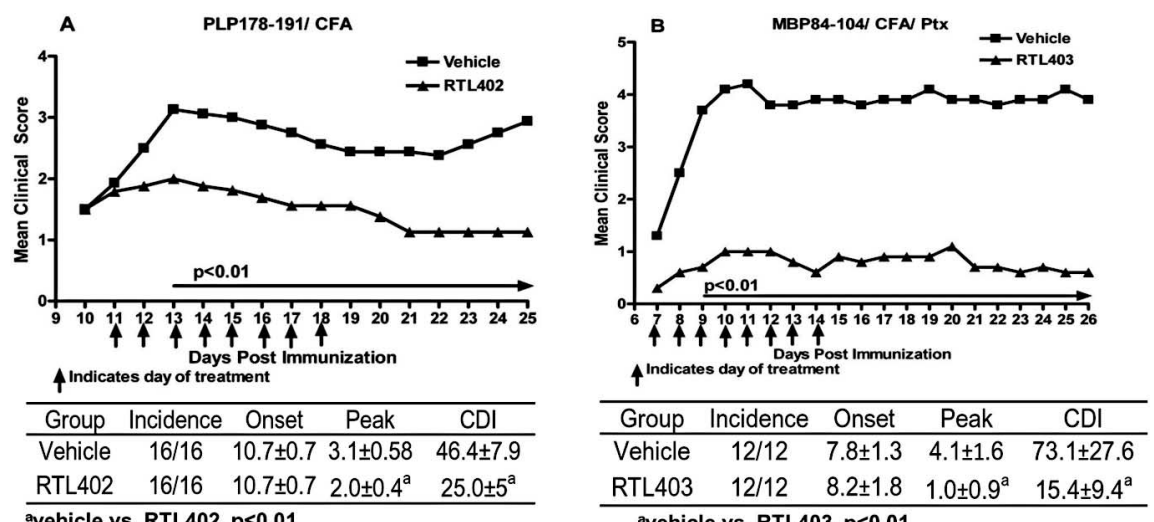

Figure 3. Two new RTLs for mice expressing I-A ${ }^{\text {s }}$, RTL402, and RTL403 containing PLP-178-191 and MBP-84-104 peptides respectively, can effectively suppress EAE induced with the cognate peptides. SJL/J mice were immunized either with PLP-178 191/CFA ( $\boldsymbol{A}$ ) or MBP-84 -104/CFA/Ptx (B). At disease onset, mice were divided into two groups and were treated with vehicle ( $\boldsymbol{A}$, B) or RTL402 (A) or RTL403 (B) subcutaneously for $8 \mathrm{~d}$ and were scored as described in Materials and Methods. Data presented are the mean of two experiments for each group, with 6-8 mice per group. Significant differences between control and RTL-treated groups were determined using the Mann-Whitney test.

the mice in the treated groups were boosted with three more RTL or vehicle injections before killing them for ex vivo analyses on day 26 after immunization.

Proliferative responses to both immunizing peptides were inhibited by a single RTL

As described above, a single RTL can suppress clinical signs of EAE induced by multiple peptides. To verify that each immunizing peptide in the mixture was immunologically active for induc- ing specific T-cells, lymph node cells from vehicle-injected mice were evaluated for proliferation responses. As shown in Figure $5, A$ and $B$, the immunization protocol that we followed did induce good proliferative responses to each of the three peptides used in this study. Overall, the mean stimulation index from vehicle-treated mice was highest $(6-10 \times)$ in response to PLP-139-151 peptide in each experiment (PLP-139-151 + PLP-178-191 and PLP139-151 + MBP-84-104 immunizations), with lower SI $(\sim 4 \times)$ in response to MBP-84-104 and PLP-178-191 peptides. The presence of proliferative responses to both the peptides used for immunizations suggests that both encephalitogenic T-cell specificities likely contributed to EAE induction, with PLP-139-151-reactive T-cells being immunodominant. Of importance, treatment of doubly immunized mice with a single RTL significantly reduced lymph node proliferative responses to both immunizing peptides (Fig. $5 A, B$ ), indicating RTL suppression of both cognate and bystander T-cells.

RTL treatment induces an antiinflammatory environment in the periphery

Mononuclear cells from spleen were stimulated with respective peptides (control group with PLP-139-151, PLP-178-191, MBP-84-104, and vehicle; RTL401 groups with PLP-139-151; RTL402 group with PLP-178-191 and RTL403 group with MBP-84-104), and culture supernatants were evaluated for levels of secreted IL-2, IL-4, IL-5, IL-6, IL-10, IFN $\gamma$, TNF $\alpha$, and IL-17. As shown in Figure 6, RTL401, RTL402, and RTL403 treatments each significantly downregulated IL-17 production and significantly upregulated production of anti-inflammatory cytokines IL-10 and IL-13 after stimulation of splenocytes with cognate peptides. None of the other studied cytokines was significantly different between the groups. These results are highly consistent with our previous data with RTL401 (Huan et al., 2004) and show the exact same pattern of cytokine deviation for RTL402 and RTL403. It is important to note that these changes in cytokine secretion were not present when cells from the RTL401-treated group were stimulated with noncognate PLP-178-191 or MBP-84-104 peptides, and the same is true for the RTL402- and RTL403-treated groups as well. This suggests that RTL treatment induces an antigen-specific immunomodulation of the targeted T-cells in the periphery, which then indirectly influence bystander T-cell responses. Cytokine production was also studied in cells isolated from CNS. Mononuclear cells from spinal cord tissue of RTL401-, RTL402-, or RTL403-treated mice produced significantly less IL-17 and IL-2 when stimulated 
with cognate peptide than did vehicletreated mice (Fig. 7). Either other cytokines were not detected or the levels were extremely low in all the groups.

Individual RTLs decrease inflammatory lesions in spinal cords of mice immunized with two peptides

Earlier, we demonstrated that RTL treatment markedly reduced CNS infiltration in mice immunized with PLP-139-151 (SJL/J) or MOG-35-55 (C57BL/6) peptides. Here we extend these findings to mice immunized with two different encephalitogenic peptides and treated with a single RTL. Histopathological examination on day 26 after immunization revealed dense focal inflammation in spinal cords of vehicle-treated mice immunized with PLP-139-151 + PLP-178-151 (Fig. $8 A)$ or PLP-139-151+ MBP-84-104 peptides (Fig. $8 \mathrm{~B}$ ), and a marked reduction of these lesions in mice treated with single RTL (Fig. 8). This observation suggests that T-cells with specificities to both immunizing peptides were reduced in the CNS of mice treated with RTL401, RTL402, or RTL403.

\section{Discussion}

Our data demonstrate for the first time that treatment with a single RTL can deviate autoimmune responses of cognate $\mathrm{T}$-cell specificities and induce bystander suppression that reverses clinical signs of EAE in mice immunized with multiple encephalitogenic peptides. We have shown previously that RTL401 (with bound PLP139-151 peptide) cannot treat EAE induced by MOG-35-55 peptide. Furthermore, RTL401 cannot treat EAE in mice immunized singly with PLP-178-191 or MBP-84-104 peptides, and the same is also true with RTL402 and RTL403. These observations point toward antigenspecific suppression of EAE by RTL modulation of the cognate T-cell specificity. However, here we provide evidence that RTL401 or RTL402 individually can suppress EAE induced with the combination of PLP-139-151 + PLP-178-191 peptides. Likewise, RTL401 or RTL403 can suppress EAE in mice immunized with the combination of PLP-139-151 + MBP84-104 peptides. PLP-139-151 was used as the common immunogen in both sets of experiments described in the current study since it is an immunodominant peptide in SJL/J mice (Anderson et al., 2000). However, we also used the combination of PLP-178-191 + MBP-84104 to induce EAE and subsequently treat with either RTL402 or RTL403. As expected, both the individual RTLs were able to suppress EAE induced with the combination of nonthymic antigens (data not shown). These results indicate that treatment with a
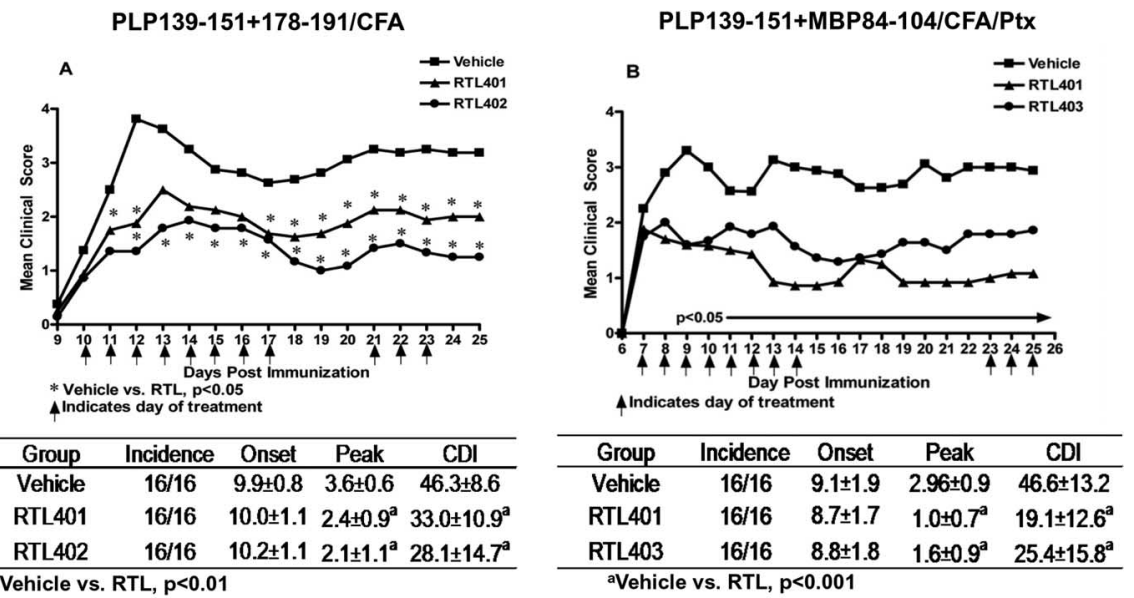

Figure 4. A single RTL was able to suppress EAE in mice immunized with two different peptides. Disease course of $S J L / J$ mice immunized with PLP-139-151 + PLP-178-191/CFA (A) and PLP-139-151 + MBP-84-104/CFA/Ptx (B). At disease onset, mice were treated daily with either vehicle (Tris HCl, pH 8.5) or with RTL401 ( $\boldsymbol{A}, \boldsymbol{B}), \mathrm{RTL} 402(\boldsymbol{A})$, or RTL403 (B) subcutaneously for $8 \mathrm{~d}$. Mice also received booster injections along with antihistamine as indicated in the figure. Data presented are the mean of two experiments for each group, with 8 mice per group. Significant differences between control and treated groups were determined using the Mann-Whitney test.
A

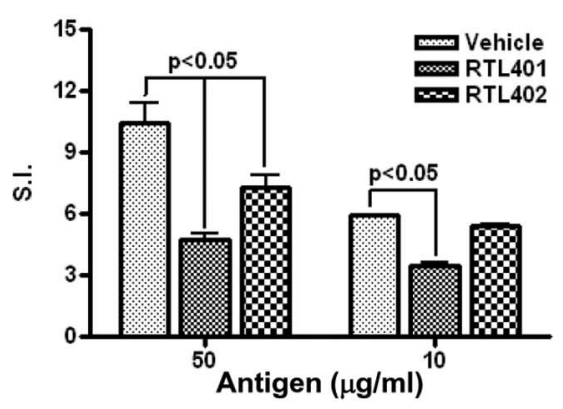

B

PLP139-151

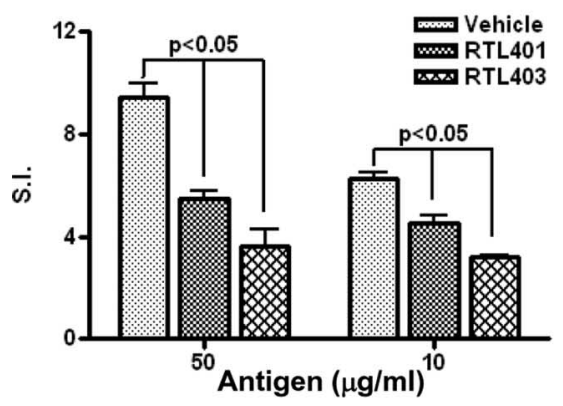

PLP178-191
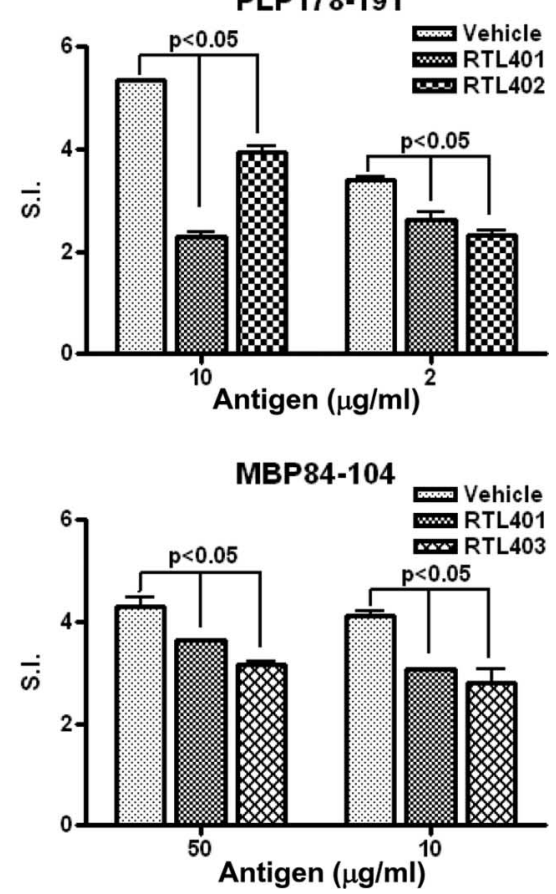

Figure 5. Proliferative response to both the immunizing peptides was present in the lymph nodes of mice immunized with PLP-139-151 + PLP-178-191 (A) or PLP-139-151 + MBP-84-104 peptides (B), with reduced proliferative response to both of the immunizing peptides after treatment with either of the cognate RTLs. Lymph nodes were isolated from vehicle- and RTL-treated groups of mice $25 \mathrm{~d}$ after immunization. The cells from four representative mice were pooled, and T-cell responses were measured by proliferation to the immunizing antigens after $72 \mathrm{~h}$ incubation in stimulation medium, the last $18 \mathrm{~h}$ in presence of ${ }^{3} \mathrm{H}$-thymidine. Data are presented as SI relative to medium alone controls and are representative of two separate experiments. Significant differences between control and treated groups were determined with Student's $t$ test.

single RTL can suppress EAE induced with multiple T-cell specificities provided that one of the immunizing peptide is present in the RTL being used for the treatment.

To address the questions of bystander suppression and cytokine deviation, we initially tested RTL treatment of EAE in mice immunized with syngenic WSCH, which would contain all the relevant myelin peptides. We used RTL401 for the treatment 


\section{Cytokine production by the splenocytes}
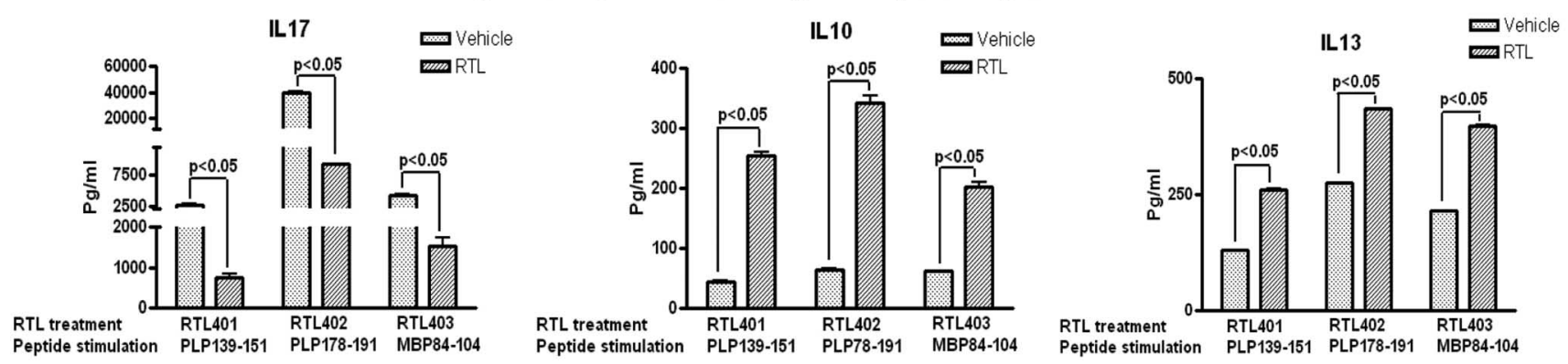

Figure 6. Cytokine production in splenocytes from dual peptide-immunized mice with EAE and treated with cognate RTL or vehicle and stimulated with the corresponding peptide in vitro. Mice were killed $25 \mathrm{~d}$ after immunization, and splenocytes from each group were cultured in vitro with $10 \mu \mathrm{g}$ of indicated peptide. Supernatants were harvested after $48 \mathrm{~h}$ and assayed for cytokine production using Bio-Plex cytokine assay kit. Data are presented as the mean \pm SD of three replicate cultures from pooled cells, and are representative of two experiments.

Cytokine production by cells isolated from CNS
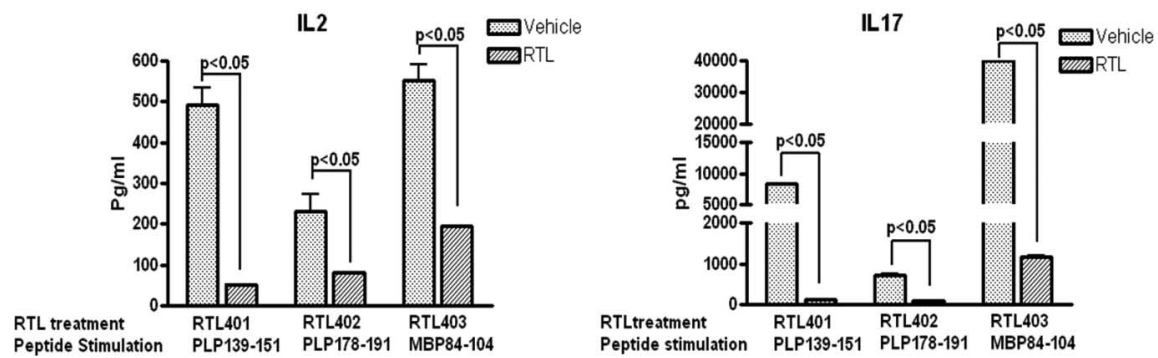

Figure 7. Cytokine production from mononuclear cells isolated from CNS of dual peptide-immunized mice with EAE treated with cognate RTL or vehicle and stimulated with the corresponding peptide in vitro. Mice were killed $25 \mathrm{~d}$ after immunization, and CNS cells from each group were cultured in vitro with $10 \mu \mathrm{g}$ of indicated peptide. Supernatants were harvested after $48 \mathrm{~h}$ and assayed for cytokine production using Bio-Plex cytokine assay kit. Data are presented as the mean \pm SD of three replicate cultures from pooled cells, and are representative of two experiments.

since PLP-139-151 is the immunodominant peptide in SJL/J mice. RTL401 was able to ameliorate clinical and histopathological signs of EAE induced with WSCH concomitant with increased production of anti-inflammatory cytokines (IL-10, IL13 , and IL-4) and reduction in proinflammatory cytokines (IL17 , and TNF $\alpha$ ). Cells from lymph nodes obtained from control mice showed proliferative responses mainly to PLP-139-151 and possibly PLP-43-64 peptides (Fig. 2), but PLP-139-151 was clearly immunodominant in WSCH-immunized mice, consistent with other reports (Whitham et al., 1996). A low frequency of T-cells responsive to other potential epitopes in WSCHimmunized mice could be the possible reason for inability to detect proliferative response to other antigens in addition to PLP139-151. This is also supported by the fact that splenocytes from vehicle-treated WSCH-immunized mice, when stimulated with PLP-178-191 and MBP-84-104 in 24 well plates, produced IL-2, IL-17, and TNF $\alpha$, suggesting the presence of T-cells responsive to these peptides in WSCH-immunized mice. Nonetheless, our data suggest that RTL treatment did modify the course of the immune response such that cells recovered from the RTL401-treated group were programmed to produce more anti-inflammatory cytokines than proinflammatory ones (e.g., a cytokine switch) when stimulated with WSCH. This observation supports the idea that the prevailing cytokine environment in the periphery of RTL-treated mice might also affect bystander T-cells with unrelated specificities.

We thus tested RTL treatment effects in mice immunized with the combination of two different encephalitogenic peptides and treated with single RTLs. Our first goal in these experiments was to establish a dual peptide-immunization protocol such that immune responses to subdominant peptides such as PLP-178-191 and MBP- $84-104$ could be detected in addition to those induced easily by the immunodominant PLP-139-151 peptide. As shown in Figure 5, $A$ and $B$, significant stimulation indices were detectable for all the three peptides used, although responses were highest to PLP-139-151 as expected. Moreover, proliferation responses were decreased to both cognate and noncognate immunizing peptides in mice treated with RTL401, RTL402, and RTL403. It is noteworthy that proliferation responses were not completely ablated to either immunizing peptide in the RTL-treated groups, indicating persistence of the targeted T-cell specificities.

To evaluate whether RTL treatment changed functional properties of the T-cells, splenocytes obtained from the different treatment groups were stimulated with respective peptides for $48 \mathrm{~h}$ and supernatants evaluated for cytokine secretion. Cells from the RTL401-, RTL402-, and RTL403-treated groups exhibited strikingly similar cytokine patterns when stimulated with PLP-139151, PLP-178-191, or MBP-84-104, respectively, each secreting significantly less IL-17 and more IL-13 and IL-10 compared with their respective controls. We previously reported reduced IL-17 levels after RTL551 treatment of MOG-35-55 induced EAE (Sinha et al., 2007). This cytokine is now recognized as the main driver of inflammation in EAE. Interestingly, when cells from the RTL401-treated group were stimulated with PLP-178-191 or MBP-84-104 (likewise for RTL402- and RTL403-treated groups) there were no differences in IL-17, IL-10, or IL-13 levels compared with controls (data not shown). This result indicates that RTL treatment causes an antigen-specific cytokine switch in the targeted encephalitogenic T-cell populations.

Of immunoregulatory significance is the increased secretion of IL-10 from splenocytes obtained from the RTL-treated groups that were stimulated with respective cognate peptides. Although initially thought to be a product of Th2 cells (Moore et al., 2001), IL-10 is also produced by B cells (Moore et al., 2001; O'Garra and Vieira, 2004), macrophages and dendritic cells (DCs) (Edwards et al., 2002; Dillon et al., 2004; Boonstra et al., 2006), mast cells, and subsets of regulatory T-cells (O'Garra and Vieira, 2004) and is known to reduce activation of macrophages and DCs (O'Garra and Vieira, 2007) as well as Th1 cell responses. Our RT-PCR 


\section{A. PLP139+151+PLP178-191/CFA}

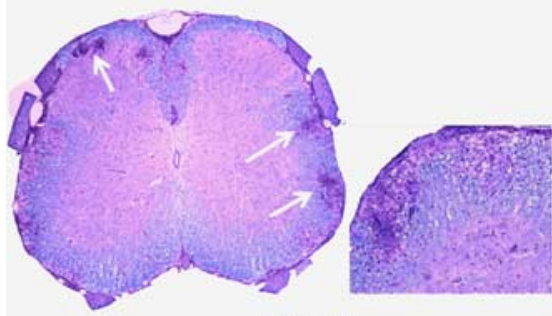

Vehicle

\section{B. PLP139+151+MBP84-104/CFA/Ptx}

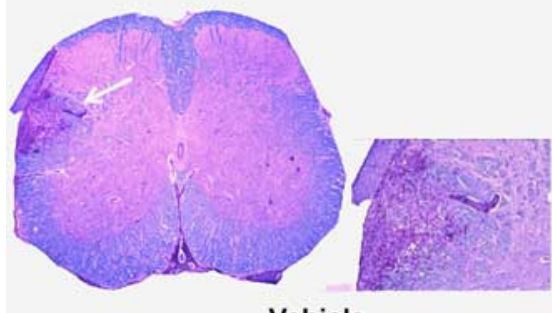

Vehicle

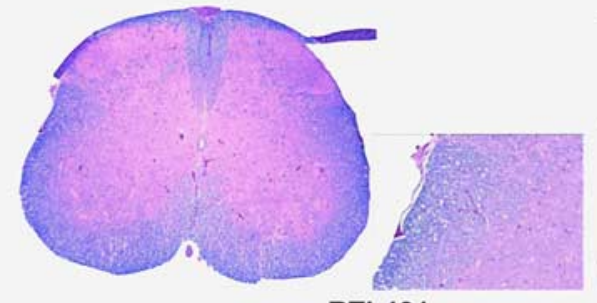

RTL401

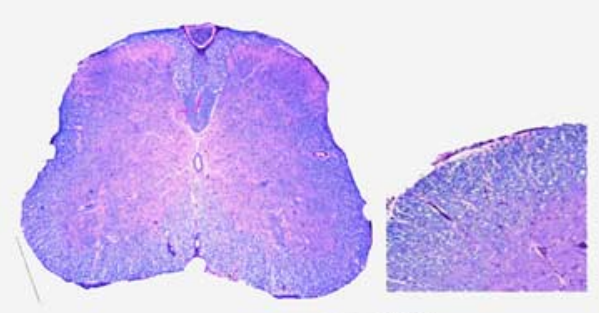

RTL401

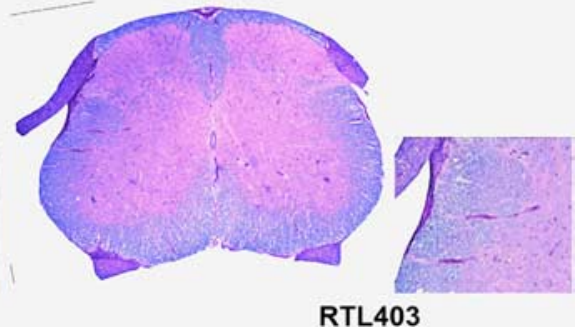

Figure 8. Fixed, paraffin-embedded spinal cord sections from vehicle- or RTL-treated SJL/J mice 25 d after immunization with PLP-139-151 + PLP-178-191/CFA (A) or PLP-139-151 + MBP-84-104/CFA/Ptx (B). Spinal cords from vehicle-treated mice $(\boldsymbol{A}, \boldsymbol{B})$ showed dense mononuclear infiltration (arrow) and loss of myelin (loss of blue staining) in the regions adjacent to the mononuclear infiltrate. Spinal cords from RTL-treated mice $(\boldsymbol{A}, \boldsymbol{B})$ showed lack of inflammatory cells with no apparent loss of myelin. Magnification: $\boldsymbol{A}, \boldsymbol{B}, 4 \times$; insets, $20 \times$.

analysis revealed that both T-cells and APCs from RTL-treated groups had significantly higher IL-10 mRNA transcripts than controls (data not shown). Moreover, our previous studies using human Th1 clones demonstrated that in vitro incubation with cognate RTL induced IL-10 secretion directly, as well as after subsequent restimulation with Ag presented by APC, indicating long-term modification of T-cell function (Burrows et al., 2001). This important anti-inflammatory cytokine induced by RTL treatment could thus mediate bystander suppression of other encephalitogenic T-cell specificities in a local environment. Similar IL-10-mediated bystander suppression has also been reported for Ig-PLP-139-151 chimeric protein used to treat EAE induced with multiple epitopes (Legge et al., 2000). Along with IL-10, RTL treatment increased production of IL-13, another anti-inflammatory cytokine.

The overall effect of this immunosuppression in the periphery correlates with a marked reduction of infiltrating cells in the spinal cords of mice treated with RTLs and decreased production on a per-cell basis of both IL-17 and IL-2 of the remaining CNSderived cells. Reduced inflammation in the CNS of mice immunized with two peptides and treated with a single RTL also suggests that migration of T-cells of both peptide specificities was affected. In contrast, spinal cords from vehicle-treated mice had infiltrating cells both in the CNS parenchyma and blood vessels.

These data lend strong support to the hypothesis that antigenspecific treatment of EAE by RTLs can induce a cytokine switch in target T-cells that likely contributes to both specific and bystander suppression in the periphery and CNS. Future studies will evaluate the minimum frequency of target $\mathrm{T}$-cells needed for RTL to trigger a cytokine switch sufficient to affect encephalitogenic T-cells of a different specificity, and whether bystander suppression affects mainly neuroantigen-specific T-cells or is simply dependent on the state of $\mathrm{T}$ activation regardless of Th subtype and TCR specificity. Our study has important implications for therapy of MS, in which little is known for certain about how many or which pathogenic T-cell specificities contribute to disease progression.

\section{References}

Anderson AC, Nicholson LB, Legge KL, Turchin V, Zaghouani H, Kuchroo VK (2000) High frequency of autoreactive myelin proteolipid proteinspecific T cells in the periphery of naïve mice: mechanisms of selection of the self-reactive repertoire. J Exp Med 191:761-770.

Boonstra A, Rajsbaum R, Holman M, Marques R, Asselin-Paturel C, Pereira JP, Bates EE, Akira S, Vieira P, Liu YJ, Trinchieri G, O’Garra A (2006) Macrophages and myeloid dendritic cells, but not plasmacytoid dendritic cells, produce IL-10 in response to MyD88- and TRIF-dependent TLR signals, and TLR-independent signals. J Immunol 177:7551-7558.

Burrows GG, Bebo BF Jr, Adlard KL, Vandenbark AA, Offner H (1998) Two-domain MHC class II molecules form stable complexes with myelin basic protein 69-89 peptide that detect and inhibit rat encephalitogenic $T$ cells and treat experimental autoimmune encephalomyelitis. J Immunol 161:5987-5996.

Burrows GG, Chang JW, Bächinger HP, Bourdette DN, Offner H, Vandenbark AA (1999) Design, engineering and production of functional single-chain T cell receptor ligands. Protein Eng 12:771-778.

Burrows GG, Chou YK, Wang C, Chang JW, Finn TP, Culbertson NE, Kim J, Bourdette DN, Lewinsohn DA, Lewinsohn DM, Ikeda M, Yoshioka T, Allen CN, Offner H, Vandenbark AA (2001) Rudimentary TCR signaling triggers default IL-10 secretion by human Th1 cells. J Immunol 167:4386-4395.

Chang JW, Mechling DE, Bächinger HP, Burrows GG (2001) Design, engineering, and production of human recombinant $\mathrm{T}$ cell receptor ligands derived from human leukocyte antigen DR2. J Biol Chem 276:24170-24176.

Chou YK, Culbertson N, Rich C, LaTocha D, Buenafe AC, Huan J, Link J, Wands JM, Born WK, Offner H, Bourdette DN, Burrows GG, Vandenbark AA (2004) T cell hybridoma specific for myelin oligodendrocyte glycoprotein $35-55$ peptide produced from HLA-DRB1* 1501 -transgenic mice. J Neurosci Res 77:670-680.

Dillon S, Agrawal A, Van Dyke T, Landreth G, McCauley L, Koh A, Maliszewski C, Akira S, Pulendran B (2004) A Toll-like receptor 2 ligand stimulates Th2 responses in vivo, via induction of extracellular signalregulated kinase mitogen-activated protein kinase and c-Fos in dendritic cells. J Immunol 172:4733-4743. 
Edwards AD, Manickasingham SP, Spörri R, Diebold SS, Schulz O, Sher A, Kaisho T, Akira S, Reis e Sousa C (2002) Microbial recognition via Tolllike receptor-dependent and -independent pathways determines the cytokine response of murine dendritic cell subsets to CD40 triggering. J Immunol 169:3652-3660.

Huan J, Subramanian S, Jones R, Rich C, Link J, Mooney J, Bourdette DN, Vandenbark AA, Burrows GG, Offner H (2004) Monomeric recombinant TCR ligand reduces relapse rate and severity of experimental autoimmune encephalomyelitis in SJL/J mice through cytokine switch. J Immunol 172:4556-4566.

Legge KL, Min B, Bell JJ, Caprio JC, Li L, Gregg RK, Zaghouani H (2000) Coupling of peripheral tolerance to endogenous interleukin 10 promotes effective modulation of myelin-activated T cells and ameliorates experimental allergic encephalomyelitis. J Exp Med 191:2039-2052.

Link JM, Rich CM, Korat M, Burrows GG, Offner H, Vandenbark AA (2007) Monomeric DR2/MOG-35-55 recombinant TCR ligand treats relapses of experimental encephalomyelitis in DR2 transgenic mice. Clin Immunol 123:95-104.

McFarland HF, Martin R (2007) Multiple sclerosis: a complicated picture of autoimmunity. Nat Immunol 8:913-919.

Moore KW, de Waal Malefyt R, Coffman RL, O'Garra A (2001) Interleukin-10 and the interleukin-10 receptor. Annu Rev Immunol 19:683-765.

Offner H, Subramanian S, Wang C, Afentoulis M, Vandenbark AA, Huan J, Burrows GG (2005) Treatment of passive experimental autoimmune encephalomyelitis in SJL mice with a recombinant TCR ligand induces IL-13 and prevents axonal injury. J Immunol 175:4103-4111.
O'Garra A, Vieira P (2004) Regulatory T cells and mechanisms of immune system control. Nat Med 10:801-805.

O'Garra A, Vieira P (2007) T(H)1 cells control themselves by producing interleukin-10. Nat Rev Immunol 7:425-428.

Sinha S, Subramanian S, Proctor TM, Kaler LJ, Grafe M, Dahan R, Huan J, Vandenbark AA, Burrows GG, Offner H (2007) A promising therapeutic approach for multiple sclerosis: recombinant T-cell receptor ligands modulate experimental autoimmune encephalomyelitis by reducing interleukin-17 production and inhibiting migration of encephalitogenic cells into the CNS. J Neurosci 27:12531-12539.

Vandenbark AA, Rich C, Mooney J, Zamora A, Wang C, Huan J, Fugger L, Offner H, Jones R, Burrows GG (2003) Recombinant TCR ligand induces tolerance to myelin oligodendrocyte glycoprotein $35-55$ peptide and reverses clinical and histological signs of chronic experimental autoimmune encephalomyelitis in HLA-DR2 transgenic mice. J Immunol 171:127-133.

Veräjänkorva E, Setälä N, Teros T, Salmi AA, Pöllänen P (2002) Testicularassociated immune deviation: flushing of the testicular lymph sinusoids induces immunosuppression and inhibits formation of EAE in SJL mice. Scand J Immunol 55:478-483.

Wang C, Mooney JL, Meza-Romero R, Chou YK, Huan J, Vandenbark AA, Offner H, Burrows GG (2003) Recombinant TCR ligand induces early TCR signaling and a unique pattern of downstream activation. J Immunol 171:1934-1940.

Whitham RH, Wingett D, Wineman J, Mass M, Wegmann K, Vandenbark A, Offner H (1996) Treatment of relapsing autoimmune encephalomyelitis with $\mathrm{T}$ cell receptor Vbeta-specific antibodies when proteolipid protein is the autoantigen. J Neurosci Res 45:104-116. 